\title{
Estudo da Hemodinâmica Fetal por meio da Dopplervelocimetria nas Artérias Umbilical e Cerebral Média Durante a Crise Hipertensiva Materna antes e após o Tratamento com Nifedipina
}

\author{
Autor: Sinval Ferreira de Oliveira
}

Orientador: Alcino Lázaro da Silva

Tese apresentada à Faculdade de Medicina da Universidade Federal de Minas Gerais, para obtenção do título de Doutor, em 11 de dezembro de 2001.

Com o objetivo de estudar a hemodinâmica fetal antes e após o tratamento da crise hipertensiva com o uso de nifedipina, foram acompanhadas 35 gestantes portadoras de hipertensão arterial crônica que desenvolveram crise hipertensiva nessa gestação (estudo tipo coorte, realizado no período de janeiro de 1995 a março de 1997). Nessas pacientes, após aferição e anotação da pressão arterial, foi avaliado o indice de resistência da artéria umbilical, a seguir o índice de resistência da artéria cerebral média (através da Dopplerfluxometria) e a freqüência cardíaca fetal. Foram administradas três gotas de nifedipina $(5 \mathrm{mg})$, por via sublingual. Após 10 minutos, as pacientes que apresentavam redução da pressão arterial para níveis de pressão arterial sistólica menor ou igual a $160 \mathrm{mmHg}$ e pressão arterial diastólica igual ou menor que 105 $\mathrm{mmHg}$ foram submetidas a outra avaliação do fluxo nas artérias umbilical e cerebral média. Repetiu-se o exame Dopplerfluxométrico nos mesmos vasos $30 \mathrm{mi}-$ nutos após o controle da pressão. As pacientes que não responderam ao tratamento inicial receberam uma segunda dose de $5 \mathrm{mg}$ de nifedipina aguardando-se mais 10 minutos. Não ocorrendo a resposta desejada, uma terceira e última dose era recomendada. Não se verificou diferença significante entre os exames Dopplerfluxométricos realizados antes do tratamento e os de 10 e 30 minutos após, em relação à variável freqüência cardíaca fetal. No que se refere ao aspecto de centralização fetal, não se observaram alterações neste item, com o tratamento hipotensor materno. Nenhum feto passou a exibir centralização de fluxo ao longo do período de controle pressórico da mãe.

Palavras-chave: Hipertensão arterial. Dopplervelocimetria. Crise hipertensiva.

\section{OPapel do Estresse Oxidativo Detectado no Líquido Folicular de Pacientes Inférteis Submetidasà Reprodução Assistida}

\author{
Autora: Eleonora Bedin Pasqualotto \\ Orientador: Prof. Dr. Vicente Mario Valentino Izzo
}

Tese de doutorado apresentada à Faculdade de Medicina Universidade de São Paulo (FMUSP), Departamento de Ginecologia, em 30 de julho de 2002.

Objetivos: Determinar a presença e os níveis de peroxidação lipídica (LPO) e capacidade antioxidante total (CAT) no líquido folicular de pacientes inférteis submetidas à reprodução assistida. Correlacionar estes niveis com maturidade oocitária, qualidade embrionária, taxas de fertilização, de clivagem e de gestação. Casuística e Métodos: Neste estudo prospectivo foram analisados 115 folículos de 41 pacientes. A captação oocitária foi guiada por ultra-sonografia transvaginal. Cada folículo foi aspirado separadamente e seu líquido não foi misturado com o meio de cultura. Gestação foi determinada pela presença de embrião intra-uterino com batimento cardíaco visto à ultra-sonografia. A LPO foi determinada pelo método do ácido tiobarbitúrico e a CAT usando um teste potencializador da quimiluminescência.
Resultados: A média dos níveis de LPO e CAT encontrados foi $0,95 \mu \mathrm{mol} \mathrm{MDA} / \mathrm{L}$ e $819,16 \mathrm{mEq}$ Trolox, respectivamente. Não houve correlação significativa entre os valores de LPO e CAT com a maturidade oocitária, taxas de fertilização, clivagem e qualidade embrionária. Ao compararmos os valores de LPO e de CAT com as taxas de gravidez, foi detectada correlação positiva $(\mathrm{r}=0,381 ; \mathrm{p}=0,014$ e $\mathrm{r}=0,522 ; \mathrm{p}=0,003$ respectivamente). Conclusões: Foi detectada correlação positiva entre os valores de LPO e CAT com as taxas de gravidez o que significa que ocorre intenso metabolismo oxidativo no folículo em desenvolvimento e que este não é prejudicial à formação e posterior implantação embrionária.

Palavras-chave: Fertilização Assistida. Folículos. Estresse Oxidativo. 